OPEN ACCESS

Edited by:

Laura Morelli,

Leloir Institute Foundation (FIL),

Argentina

Reviewed by:

Maria Carolina Dalmasso,

Leloir Institute Foundation (FIL),

Argentina

Elena Tamagno,

University of Turin, Italy

*Correspondence:

Hong-ping Chen

chen_hongping@126.com

You-ping Liu

719281681@qq.com

Received: 06 December 2018

Accepted: 28 March 2019

Published: 17 April 2019

Citation:

You Q, Li L, Xiong S, Yan Y, Li D, Yan

N, Chen H and Liu Y (2019)

Meta-Analysis on the Efficacy and Safety of Hyperbaric Oxygen as Adjunctive Therapy for Vascular

Dementia.

Front. Aging Neurosci. 11:86 doi: 10.3389/fnagi.2019.00086

\section{Meta-Analysis on the Efficacy and Safety of Hyperbaric Oxygen as Adjunctive Therapy for Vascular Dementia}

\author{
Qiang You ${ }^{1,2}$, Lan $\mathrm{Li}^{3}$, Su-qin Xiong ${ }^{1}$, Yu-fen Yan ${ }^{1}$, Dan $\mathrm{Li}^{1}$, Na-na Yan ${ }^{1}$, Hong-ping Chen ${ }^{1 *}$ \\ and You-ping Liu ${ }^{1 *}$ \\ 'Standardization Education Ministry Key Laboratory of Traditional Chinese Medicine, Department of Pharmacy, Chengdu \\ University of Traditional Chinese Medicine, Chengdu, China, ${ }^{2}$ The Affiliated Hospital, Southwest Medical University, Luzhou, \\ China, ${ }^{3}$ Department of Nursing, Southwest Medical University, Luzhou, China
}

Background: Vascular dementia (VD) is a common type of disease in the elderly. Numerous clinical trials have suggested that hyperbaric oxygen is an effective and safe complementary therapy for aging-related disorders. However, there is no reliable systematic evidence regarding hyperbaric oxygen therapy (HBOT) for the treatment of VD. Therefore, we performed a meta-analysis to evaluate the clinical efficacy and safety of HBOT in treating VD.

Methods: We methodically retrieved the clinical studies from eight databases (PubMed, Cochrane Library, Embase, Web of Science, Sino-Med, China National Knowledge Infrastructure (CNKI), China Science and Technology Journal Database (VIP), and WanFang) from their inception to November 2018. RevMan 5.3.5 was used for quality assessment and data analysis. Stata 15.1 was employed for publication bias detection and sensitivity analysis.

Results: Twenty-five randomized clinical trials (RCTs) involving 1,954 patients met our inclusion criteria. These articles researched the HBOT + oxiracetam + conventional therapy (CT) vs. oxiracetam + CT $(n=13)$, HBOT + butylphthalide $+\mathrm{CT}$ vs. butylphthalide + CT $(n=5)$, HBOT + donepezil + CT vs. donepezil + CT $(n=4)$, HBOT + nicergoline + CT vs. nicergoline + CT $(n=2)$ and HBOT + CT vs. CT $(n=1)$. The results indicated that additional HBOT strikingly improved the Mini-Mental State Examination (MMSE) ( $M D=4.00 ; 95 \% \mathrm{Cl}=3.28-4.73 ; P<0.00001)$, activities of daily living (ADL) $(M D=-5.91 ; 95 \% \mathrm{Cl}=-6.45,-5.36 ; P<0.00001)$ and $\mathrm{ADL}$ by Barthel index (BADL) $(M D=13.86 ; 95 \% \mathrm{Cl}=5.63-22.10 ; P=0.001)$ and increased the total efficacy rate (TEF) $(O R=4.84,95 \% \mathrm{Cl}=3.19-7.33, P<0.00001)$. The adverse events rates were not statistically significant between the HBOT and CT groups (OR $=0.85$, $95 \% \mathrm{Cl}=0.26-2.78, P=0.79$ ). 
Conclusion: In view of the effectiveness and safety of HBOT, the present meta-analysis suggested that HBOT can be recommended as an effective and safe complementary therapy for the treatment of $\mathrm{VD}$.

Protocol Registration: PROSPERO (ID: CRD42019117178). Available online at: http:// www.crd.york.ac.uk/PROSPERO/display_record.asp?ID=CRD42019117178.

Keywords: hyperbaric oxygen therapy, complementary therapy, systematic review, meta-analysis, vascular dementia

\section{INTRODUCTION}

Vascular dementia (VD), with clinical manifestations of cognitive disorder, cerebrovascular pathologies and progressive memory decline (O'Brien and Thomas, 2015), leads to more than $20 \%$ of all aphronesia cases worldwide and is second only to Alzheimer's disease (AD) (Gorelick et al., 2011). In particular, VD mainly affects patients in developing countries due to poorer health care and control of cardiovascular risk factors. It has been reported that in certain Asian countries, the prevalence of VD in 65-yearolds is $0.6-2.1 \%$ (Kalaria et al., 2008). Considering the rapid aging of the global population, especially in Asia (Ferri et al., 2005), and the increasing incidence rate of cardiovascular disease, there will be a tremendous increase in the morbidity of VD among the elderly within the next few decades (Barker et al., 2014; Etherton-Beer, 2014).

The main risk factors include hypertension, obesity, diabetes, hyperlipidemia, metabolic syndromes, dyslipidemia, cardiac diseases, smoking, hyperhomocysteinemia and genetic disposition (Levine and Langa, 2011; Sahathevan et al., 2012; Yates et al., 2012; Hasnain and Vieweg, 2014). Transient ischemic attack caused by stroke or acute cerebral infarction, which does enormous damage to cerebral vessels, is regarded as the major pathogenic factor of VD (Kalaria et al., 2016), and cerebrovascular diseases (CVDs) leading to lower cognitive performance for both $\mathrm{VD}$ and $\mathrm{AD}$, and this has been widely accepted (O'Brien and Markus, 2014). VD patients not only endure lower quality of life, psychological and physical harm but also pose significant medical and financial burdens on families and society. Thus, the research into effective treatment of VD is of great social and clinical significance.

Currently, conventional therapy (CT), including anticoagulant drugs, dilating cerebral vessels, reducing blood viscosity, correcting electrolyte disorders and controlling cerebral edema, has been mostly concentrated on symptomatic management and reduction of underlying risk factors for cerebrovascular disease (Sorrentino et al., 2008). The cholinesterase inhibitors (donepezil, rivastigmine, and galantamine) and non-cholinergics (memantine, nimodipine, and hydergine) have been considered effective drugs for VD (Chen et al., 2011; Jellinger, 2014). However, because of the frequent contraindications, side effects and the unclear mechanism of its pathology, the effect of CT remained limited. Recently, complementary therapy that can substantially improve the disease has been proposed.
Hyperbaric oxygen therapy (HBOT) as an adjuvant treatment with the curative administration of $100 \%$ oxygen in an elevated pressure environment of more than 1.4 atmosphere absolute (García-Covarrubias and Cuauhtémoc Sánchez-Rodríguez, 2000) has shown therapeutic effects in the treatment of VD. A typical treatment consists of $100 \%$ oxygen at $0.2 \mathrm{MPa}$ for 60-120 min (Sanchez, 2013). According to the result of previous studies, the possible mechanism of HBOT in treating VD mainly comprises: increasing oxygen supply, raising the oxygen partial pressure of the tissue, decreasing intracranial pressure, relieving brain edema, promoting tissue healing and angiogenesis, improving metabolism, reducing apoptosis, alleviating oxidative stress, increasing mitochondrial function and promoting cell differentiation and regeneration (Robertson and Hart, 1999; Feldmeier and Undersea and Hyperbaric Medical Society, 2003; Wang et al., 2016). Though not fully understood, the efficacy and safety of HBOT was indisputable. A 2012 Cochrane review performed by Xiao et al tried to assess the effectiveness and safety of HBOT for VD. However, they failed to provide sufficient and reliable clinical evidence for HBOT in treating VD because only one randomized controlled trial involving 64 patients was included in their system review (Xiao et al., 2012). Therefore, it is necessary to conduct a meta-analysis again by collecting more clinical data to evaluate the efficacy and safety of HBOT for VD.

\section{METHODS}

\section{Search Strategy}

The Cochrane library, Web of science, PubMed, Embase, CNKI (China National Knowledge Infrastructure), Wan-Fang, VIP (Chinese Science and Technology Periodical Database), and Sino-Med (Chinese Biomedical) were systematically searched from inception to November 2018. To obtain the maximum possible number of RCTs, we searched the above four commonly used Chinese databases. In brief, we used the following search strategies: subject terms + entry terms: (1) [Title/abstract] ("Hyperbaric oxygen" OR "Hyperbaric Oxygenation" $O R$ "HBOT" $O R$ "Oxygen Therapy" $O R$ "High pressure oxygen" OR "HPO"); (2) Title/abstract: ("dementia*” $O R$ "aphronesia*" $O R$ "Amentia*" $O R$ "VD" $O R$ "VaD"). Then, (1) and (2) were connected with "AND." In terms of the Chinese databases, we used the following key words: Gaoyayang [Title/Abstract] and Chidai [Title]. The search results were imported to document management software for the further screening. 


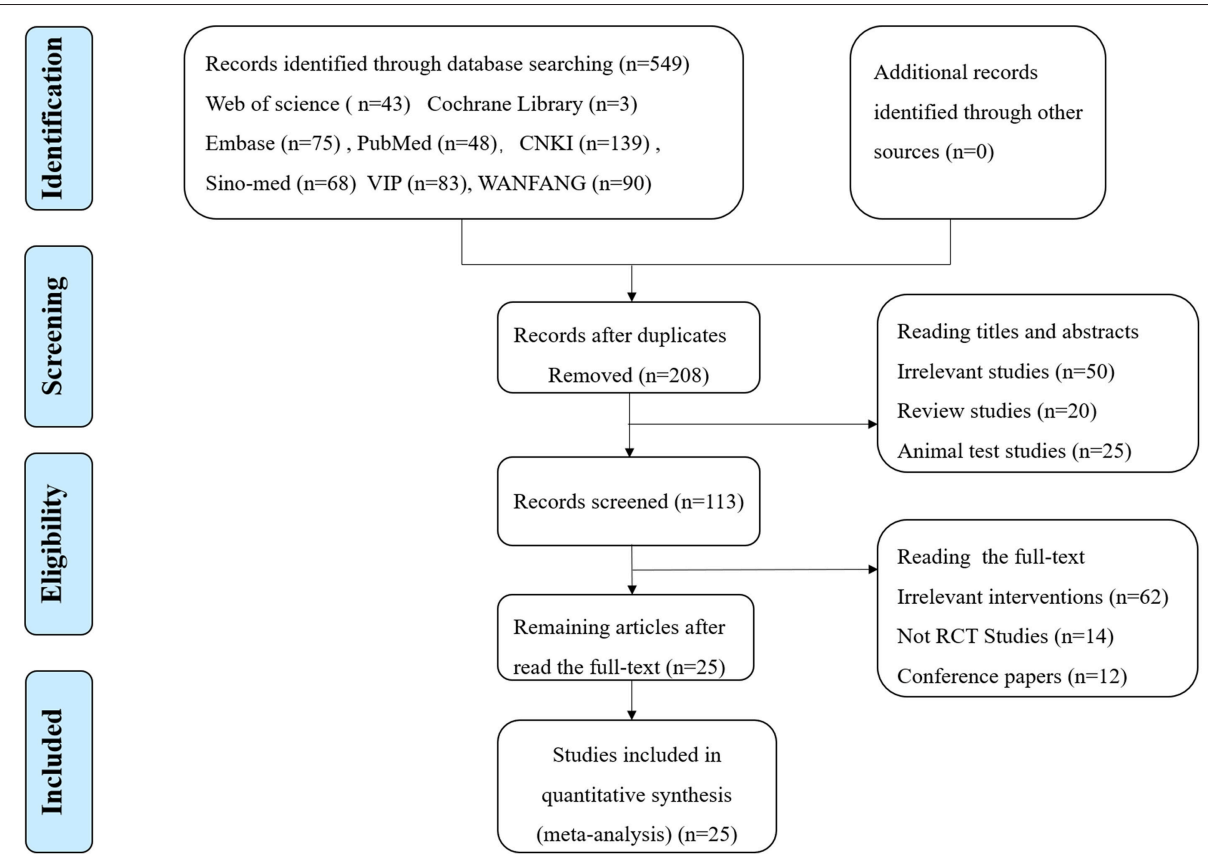

FIGURE 1 | The flowchart of study selection.

\section{Study Selection and Data Extraction}

Inclusion criteria: (1) if the study was an RCT performed in humans, whether they were blinded or not. (2) Patients were diagnosed with VD according to "Diagnostic and statistical manual of mental disorders DSM-IV (Christopher, 1994)," issued by American Psychiatric Association (APA), or "Draft diagnostic criteria for VD (DDC-VD)" (Qian et al., 2002) published by Neurological branch of Chinese Medical Association, or other accepted diagnostic criteria for VD. (3) The experimental group was treated with HBOT and conventional therapy (CT), and the control group was treated with conventional therapy without regard to the treatment duration, age, course of disease, sex, and ethnicity. (4) At least one or more outcome indicator, including MMSE, ADL, BADL and TEF, was applied to evaluate the curative effect. Exclusion criteria: (1) Non-RCTs, (2) animal experiments, (3) systematic review and case reports, (4) incorrect or incomplete data, and (5) conventional treatment was inconsistent between the control group and the experimental group. Two reviewers (Nana Yan and Dan Li) independently extracted the data of the literature, including the following contents: general trial characteristics (first author's last name, publication date, study period and diagnostic criteria); baseline patient and disease data (number of patients in each group and age); interventions (HBOT name, Western medicine name, treatment duration and dose), outcome definitions, and detailed adverse reactions. Discrepancies were resolved by consensus or a third researcher.

\section{Quality Assessment}

Two reviewers (Yufen Yan and Suqin Xiong) independently assessed the methodological qualities of the trials in accordance with the Cochrane manual delineated in version 5.3.5. The risk of bias consisted of seven items: selection bias, performance bias, detection bias, attrition bias, reporting bias, and other bias. Each item was classified into low bias risk, high bias risk, and unclear bias risk. Disagreements between reviewers were settled through discussion.

\section{Statistical Analysis}

In this review, the statistical analyses were conducted by reviewer manager (version 5.3.5), and OR presented with $95 \%$ confidence intervals $(C I)$ (employed for the analyses of dichotomous data), whereas the continuous data were presented as $M D$ with $95 \%$ CI. A fixed-effects and random- effects model was used to merge the data according to heterogeneity, which was determined using the chi-square test. With the $I^{2}$ statistic, an $I^{2}<25 \%$, indicates that heterogeneity may not be important, a value between 25 and $50 \%$ represents moderate inconsistency, and $I^{2}>50 \%$ suggest severe heterogeneity. We defined $P \geq 0.1$ and $I^{2}<50$ as an indicator that the results have good agreement and that the fixed-effects model (FEM) may be set, while $I^{2}>50 \%$ was defined as an indicator of striking heterogeneity between the data. Then, a random-effects model was employed to pool the results to minimize the influence of potential clinical heterogeneity. Stata 15.1 was used for detection of the possible sources of significant heterogeneity by sensitivity analysis. Publication bias was detected by Egger's test. $P<0.05$ suggested that there was publication bias. Subgroup analysis was conducted to assess differential associations between studies based on: (1) HBOT + CT + oxiracetam vs. CT + oxiracetam, (2) HBOT + CT + butylphthalide vs. CT + butylphthalide, (3) HBOT $+\mathrm{CT}+$ donepezil vs. CT + donepezil, (4) HBOT $+\mathrm{CT}+$ nicergoline vs. $\mathrm{CT}+$ nicergoline, and (5) HBOT $+\mathrm{CT}$ vs. CT. 


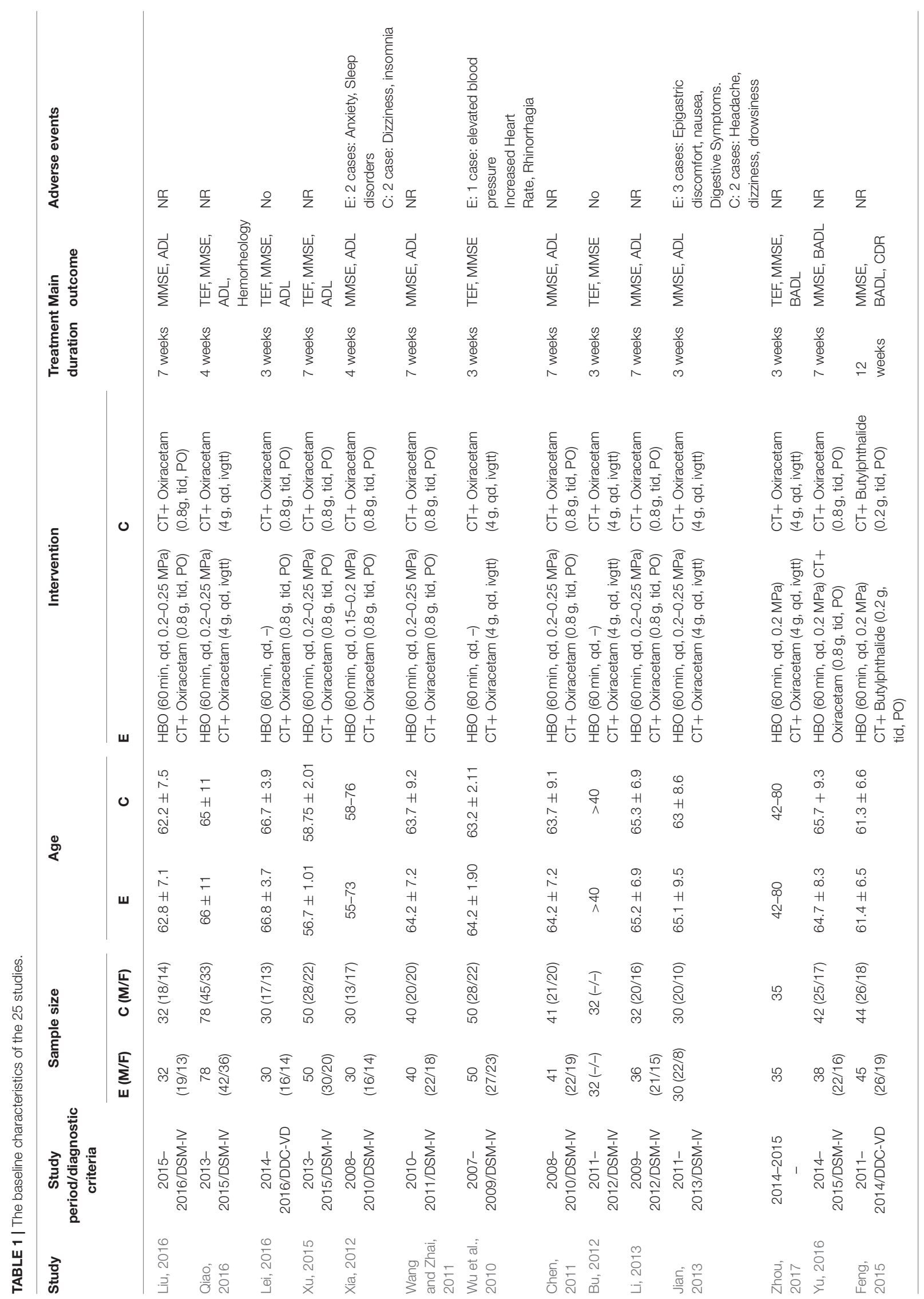




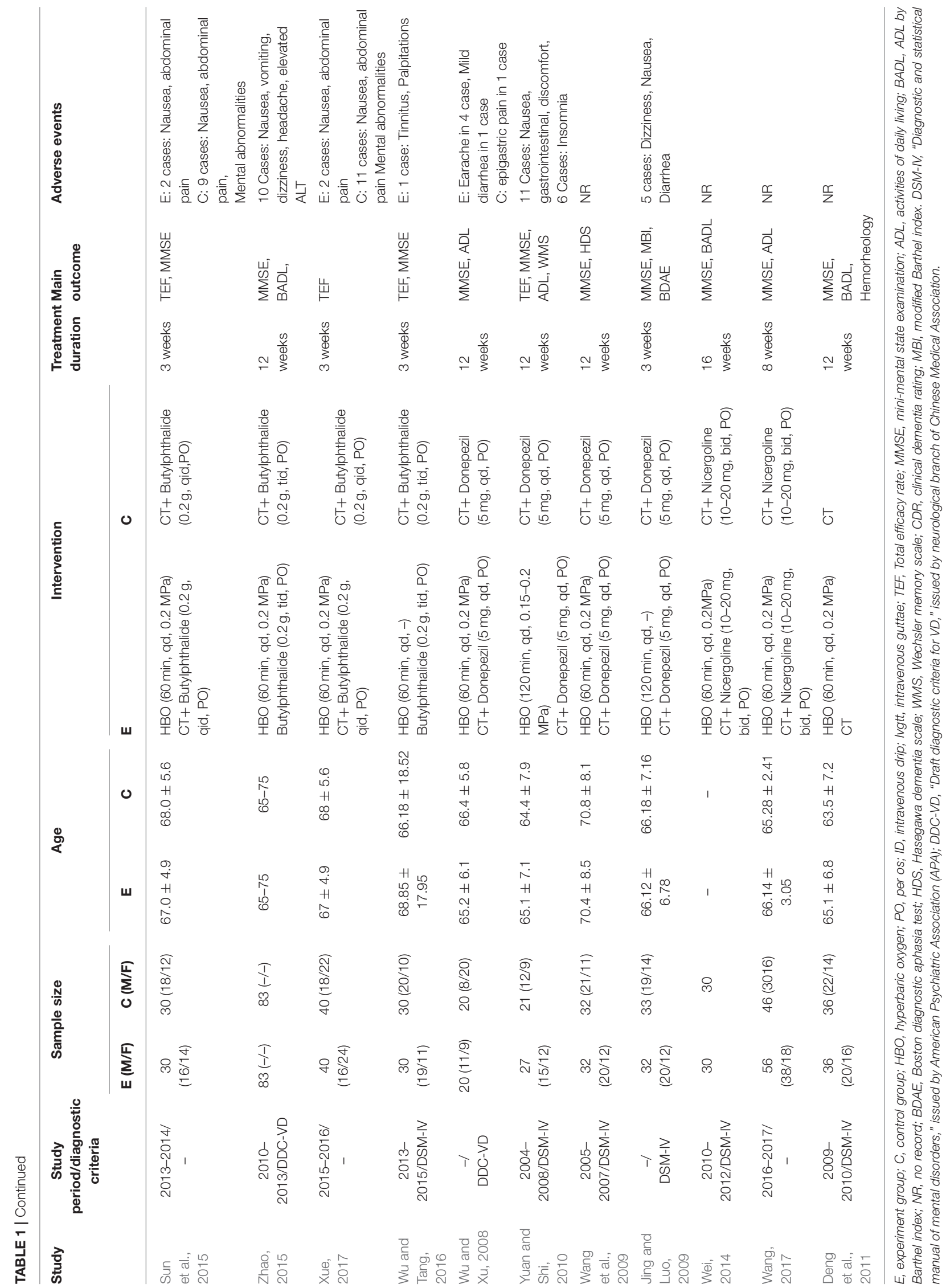


TABLE 2 | The meta-analysis result of hemorheology.

\begin{tabular}{|c|c|c|c|c|c|c|}
\hline \multirow[t]{2}{*}{ Secondary outcome } & \multirow[t]{2}{*}{$95 \% \mathrm{Cl}$} & \multirow[t]{2}{*}{$P$-value } & \multirow[b]{2}{*}{$\chi^{2}$} & \multicolumn{2}{|c|}{ Study heterogeneity } & \multirow[b]{2}{*}{$P$-value } \\
\hline & & & & df & $I^{2}, \%$ & \\
\hline Plasma viscosity & $M D-0.27[-0.53,-0.00]$ & 0.05 & 23.18 & 1 & 96 & $<0.00001$ \\
\hline Hematocrit value & $M D-0.03[-0.04,-0.02]$ & $<0.0001$ & - & - & - & - \\
\hline Erythrocyte sedimentation rate & $M D-3.30[-6.13,-0.48]$ & 0.02 & 6.44 & 1 & 84 & 0.01 \\
\hline Fibrinogen & $M D-0.30[-0.45,-0.16]$ & $<0.0001$ & 1.79 & 1 & 44 & 0.18 \\
\hline
\end{tabular}

TABLE 3 | Subgroup analysis for oxygen intake and treatment duration.

\begin{tabular}{|c|c|c|c|c|c|}
\hline Subgroups & Trials & Effects model & Pooled effect & $95 \% \mathrm{Cl}$ & $P$-value \\
\hline \multicolumn{6}{|l|}{ TEF } \\
\hline Treatment duration (3-4weeks) & 7 & Fixed & OR 3.56 & $2.11,6.00$ & $<0.00001$ \\
\hline Treatment duration (7-8weeks) & 1 & Fixed & OR 12.31 & $3.38,44.89$ & 0.0001 \\
\hline Treatment duration (12-16weeks) & 2 & Fixed & OR 6.77 & $2.91,15.74$ & $<0.00001$ \\
\hline Total & 10 & Fixed & 4.84 & $3.19,7.33$ & $<0.00001$ \\
\hline \multicolumn{6}{|c|}{ Test for subgroup differences: $\mathrm{Chi}^{2}=3.93 . \mathrm{df}=2(P=0.14) . l^{2}=49.1 \%$} \\
\hline \multicolumn{6}{|l|}{ MMSE SCORE } \\
\hline Oxygen intake (60 min, qd) & 22 & Random & MD 4.10 & $3.35,4.85$ & $<0.00001$ \\
\hline Oxygen intake (120 min, qd) & 2 & Random & MD 3.04 & $-1.04,7.12$ & 0.14 \\
\hline Total & 24 & Random & MD 4.00 & $3.28,4.73$ & $<0.00001$ \\
\hline \multicolumn{6}{|c|}{ Test for subgroup differences: $\mathrm{Chi}^{2}=0.25 . \mathrm{df}=1(P=0.62) \cdot \mathrm{R}^{2}=0 \%$} \\
\hline Treatment duration (3-4weeks) & 10 & Random & MD 3.42 & $2.28,4.45$ & $<0.00001$ \\
\hline Treatment duration (7-8weeks) & 7 & Random & MD 5.53 & $4.73,6.33$ & $<0.00001$ \\
\hline Treatment duration (12-6weeks) & 7 & Random & MD 3.37 & $2.65,4.08$ & $<0.00001$ \\
\hline Total & 24 & Random & MD 4.00 & $3.28,4.73$ & $<0.00001$ \\
\hline \multicolumn{6}{|c|}{ Test for subgroup differences: $\mathrm{Chi}^{2}=17.64 \mathrm{df}=2(P=0.0001) \cdot l^{2}=88.7 \%$} \\
\hline \multicolumn{6}{|l|}{ ADL SCORE } \\
\hline Treatment duration (3-4weeks) & 4 & Fixed & $M D-5.42$ & $-6.43,-4.40$ & $<0.00001$ \\
\hline Treatment duration (7-8weeks) & 6 & Fixed & MD -6.13 & $-6.78,-5.48$ & $<0.00001$ \\
\hline Treatment duration (12-6weeks) & 2 & Fixed & MD -5.30 & $-8.99,-1.62$ & 0.005 \\
\hline Total & 12 & Fixed & $\mathrm{MD}-5.91$ & $-6.45 \cdot-5.36$ & $<0.00001$ \\
\hline \multicolumn{6}{|c|}{ Test for subgroup differences: $\mathrm{Chi}^{2}=1.44 . \mathrm{df}=2(P=0.49) . I^{2}=0 \%$} \\
\hline \multicolumn{6}{|l|}{ ADVERSE EVENTS } \\
\hline Treatment duration (3-4weeks) & 8 & Random & OR 0.58 & $0.19,1.77$ & 0.34 \\
\hline Treatment duration (12-16weeks) & 1 & Random & OR 0.63 & $0.67,60.16$ & 0.11 \\
\hline Total & 9 & Random & OR 0.85 & $0.26,2.78$ & 0.79 \\
\hline \multicolumn{6}{|c|}{ Test for subgroup differences: $\mathrm{Chi}^{2}=3.49 . \mathrm{df}=1(P=0.06) . \mathrm{R}^{2}=71.3 \%$} \\
\hline
\end{tabular}

\section{RESULTS}

\section{Study Inclusion and Characteristics}

Of the 549 potentially relevant studies searched from the eight databases, 341 duplicated publications were removed, and 208 papers were left for further screening. After reading titles and abstracts, there were 113 reports left, and 87 articles were excluded for one of following reasons: (1) irrelevant study, (2) not a randomized controlled trial, (3) conference papers. Finally, the remaining 25 RCTs with a total of 1.954 patients meeting our inclusion criteria (983 for HBOT group and 971 for control group) were included in the final review (Figure 1). Additionally, all trials were performed and published in China. Among the 25 studies, five comparisons were employed between the HBOT group and the control group, including $\mathrm{HBOT}+\mathrm{CT}+$ oxiracetam vs. $\mathrm{CT}+$ oxiracetam (13 RCTs), HBOT+CT+ butylphthalide vs. CT+ butylphthalide (5 RCTs), HBOT+ CT+ donepezil vs. $\mathrm{CT}+$ donepezil (4 RCTs), HBOT $+\mathrm{CT}+$ nicergoline vs. $\mathrm{CT}$ + nicergoline (2 RCTs), and HBOT+CT vs. CT (1 RCT). The treatment duration lasted from 3 to 16 weeks, and sample sizes varied from 40 to 156 . Twelve studies reported specific adverse events (Table 1).

\section{Methodological Quality Assessment}

Among the 25 included trials, three studies (Feng, 2015; Liu, 2016; Qiao, 2016) applied random number tables for random sequence generation. Therefore, we considered them to be low 
risk. Five trials (Jian, 2013; Wei, 2014; Lei, 2016; Yu, 2016; Wang, 2017) were high risk due to using non-standard randomized methods, including registration order, odd-even sequence of the case caudal numbers or therapeutic regimen, while the remaining 17 studies did not offer any detailed information regarding the generation of random sequence. Almost all the studies failed to give the specific allocation concealment, performance bias and detection bias. In terms of incomplete outcome data, one study (Xue, 2017) was at high risk for the absence of a detailed MMSE score. Overall, the quality of the article is relatively low or remained indistinct because the unclear risk of biases took up a large proportion in their research. The particular results of bias assessment are summarized in Figure 2.

\section{Primary Outcomes}

\section{TEF: Addition of HBOT vs. Conventional Therapy}

Ten studies involving 798 (40.8\%) participants reported the TEF based on the MMSE score, with 402 (40.9\%) patients randomized to receive additional HBOT and $396(40.8 \%)$ patients randomized to receive $\mathrm{CT}$. No heterogeneity was observed after the heterogeneity test $\left(P=0.59, \chi^{2}=0.42, I^{2}=0 \%\right)$, and the fixed-effects model was selected for merging the data. The results showed a statistically significant difference between the HBOT and control groups, which suggested that the treatment of VD with addition of HBOT was better than routine treatment in terms of the TEF (OR $=4.84,95 \% \mathrm{CI}=3.19-7.33, P<0.00001)$, and no differences existed by subgroup analysis $(P=0.8$, $\left.I^{2}=0 \%\right)$, as shown in Figure 3 .

\section{MMSE Score: Addition of HBOT vs. \\ Conventional Therapy}

Of the included articles, all the studies except one (Xue, 2017) employed the MMSE to evaluate the addition of HBOT in the improvement of curative effects. Considering the significant heterogeneity $\left(P<0.00001, I^{2}=83 \%\right)$, we synthesized the data using a random-effects modal and introduced subgroup analysis including (1) HBOT + oxiracetam + CT vs. oxiracetam + CT $(n=13)$, HBOT + butylphthalide + CT vs. butylphthalide + CT $(n=4)$, HBOT + donepezil + CT vs. donepezil + CT $(n=4), \mathrm{HBOT}+$ nicergoline $+\mathrm{CT}$ vs. nicergoline $+\mathrm{CT}$ $(n=2)$ and HBOT + CT vs. CT $(n=1)$. The pooled results strikingly favored the HBOT $+\mathrm{CT}$ group $(M D=4.00 ; 95 \% C I$ $=3.28-4.73 ; P<0.00001)$. Subgroup analysis showed that no statistically significant difference was observed among the five groups $\left(P=0.1, I^{2}=48 \%\right)$, and the heterogeneity mainly comes from the group treated with oxiracetam $(M D=4.71 ; 95 \% C I$ $\left.=3.45-5.97 ; P<0.00001 ; I^{2}=90 \%\right)$. Sensitivity analyses were further conducted with Stata15.1 software to find the potential sources of heterogeneity. The results showed that one trial (Qiao, 2016) increased overall heterogeneity by 10 percent. In short, the MMSE of the HBOT group can be improved more effectively than that of the CT group as shown in Figure 4.

\section{ADL Score: Addition of HBOT vs. Conventional Therapy}

There were 12 RCTs with a total of 924 (47.2\%) patients recording the ADL score. Meta-analysis indicated that patients in the

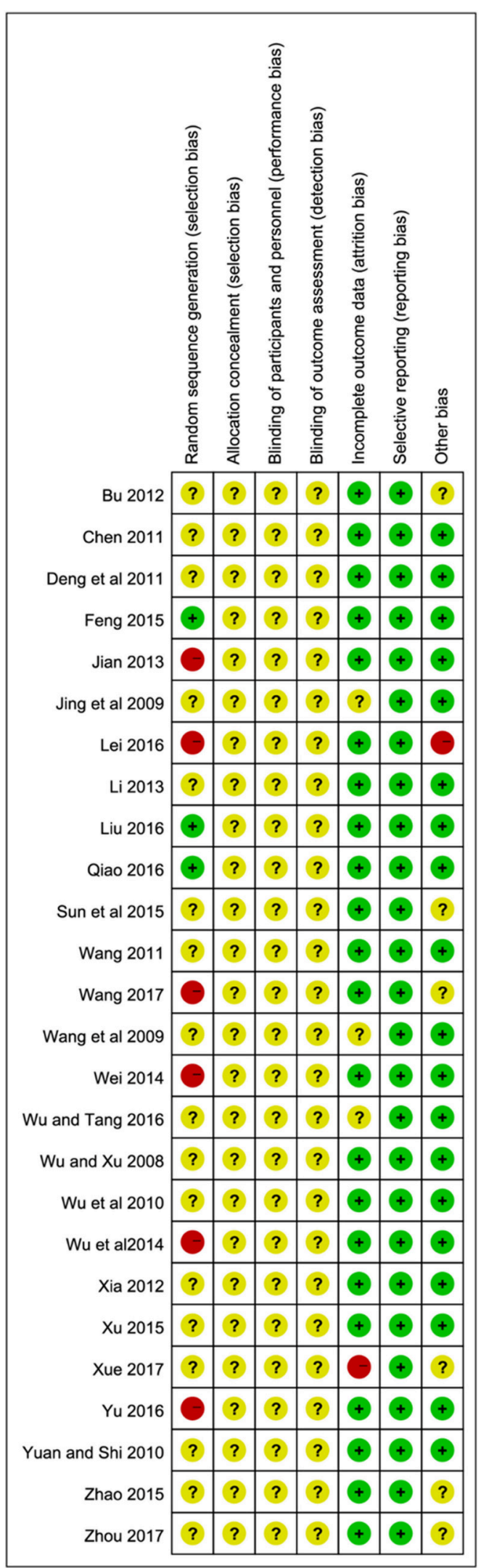

FIGURE 2 | Risk of bias assessment of the 25 trials. 


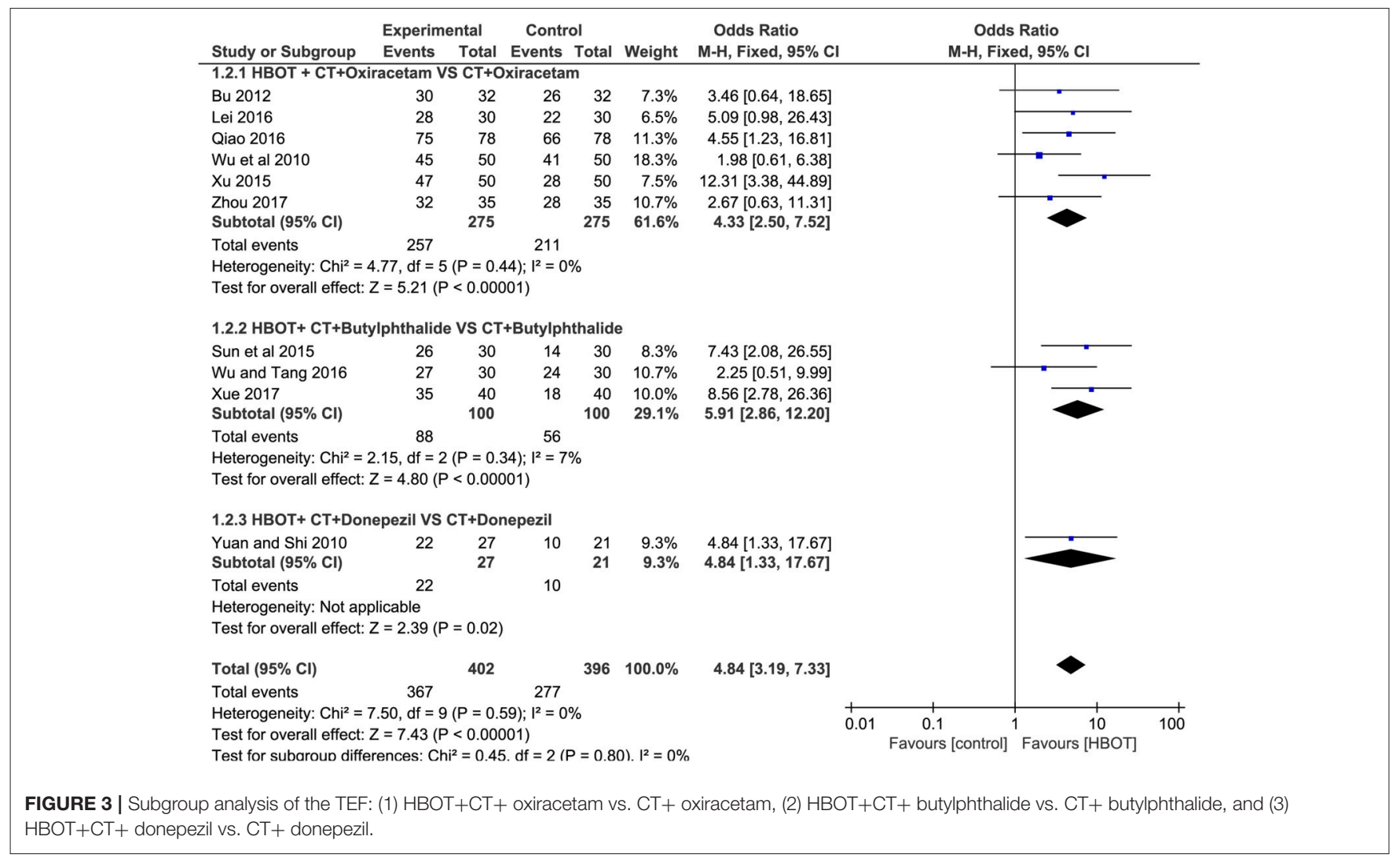

HBOT ( $n=470,47.8 \%$ ) group benefit more than those in the conventional therapy group $(n=454,46.8 \%)$ in terms of ADL score. $(M D=-5.91 ; 95 \% C I=-6.45$ to $-5.36 ; P<0.00001$; heterogeneity: $P=0.52, \chi^{2}=10.11, I^{2}=0 \%$ ), as shown in Figure 5.

\section{BADL Score: Addition of HBOT vs. Conventional Therapy}

The BADL was measured with the Barthel index method in six other trials, including Deng et al. (2011), Wei (2014), Feng (2015), Zhao (2015), Yu (2016), and Zhou (2017). The results showed the participants in the HBOT group ( $n=267,27.2 \%)$ had a statistically significant higher BADL score than patients in the conventional group $(n=270,27.8 \%)$ but with substantial heterogeneity $(M D=13.86$; $95 \% C I=5.63-22.10 ; P=0.001$; heterogeneity: $\left.P<0.00001, \chi^{2}=175.49, I^{2}=97 \%\right)$. After sensitivity analyses by removing two articles (Wei, 2014; Zhao, 2015), no heterogeneity was observed and the outcomes of BDAL between the HBOT group $(n=154,15.7 \%)$ and control group $(n=157,15.9 \%)$ remained stable $(M D=11.93 ; 95 \% C I=9.24-$ 14.63; $P<0.00001$; heterogeneity: $\left.P=0.94, \chi^{2}=0.42, I^{2}=0 \%\right)$, as shown in Figure 6.

\section{Secondary Outcomes}

Hemorheology: Addition of HBOT vs.

\section{Conventional Therapy}

The specific indicators of Hemorheology, including plasma viscosity, hematocrit value, erythrocyte sedimentation rate (ESR) and fibrinogen, were reported in two documents (Deng et al., 2011; Qiao, 2016), with 114 patients in each group. In comparison with the CT group, the HBOT group showed a greater reduction in the four indicators of Hemorheology, as shown in Figure 7 and Table 2.

\section{Adverse Events}

Adverse events were reported in 12 trials ( $\mathrm{Wu}$ and $\mathrm{Xu}, 2008$; Jing and Luo, 2009; Wu et al., 2010; Yuan and Shi, 2010; Bu, 2012; Xia, 2012; Jian, 2013; Sun et al., 2015; Zhao, 2015; Lei, 2016; Wu and Tang, 2016; Xue, 2017). Three of them (Jing and Luo, 2009; Yuan and Shi, 2010; Zhao, 2015), with a total of 279 patients, recorded the specific adverse events, including nausea, vomiting, dizziness headache, insomnia, and elevated alanine transaminase $(23 / 279,11.5 \%)$, but they failed to identify which group the adverse reactions were associated with. Therefore, adverse events (HBOT: 16/292, 5.5\%; CT: 25/292, 8.6\%) with adequate information were detailed in nine studies, and two of them (Bu, 2012; Lei, 2016) declared there were no adverse events in their trial. The most frequent adverse reactions mentioned in these studies were gastrointestinal discomfort symptoms (nausea, diarrhea and abdominal pain) and dizziness, anxiety, insomnia and drowsiness. One trial (Wu et al., 2010) reported 1 case with elevated blood pressure, increased heart rate and rhinorrhagia in HBOT group. Another article (Wu and Tang, 2016) reported 1 patient with tinnitus and palpitations in the experimental group. In addition, patients experienced mental abnormalities in two studies (Sun et al., 2015; Xue, 2017). In general, all the 


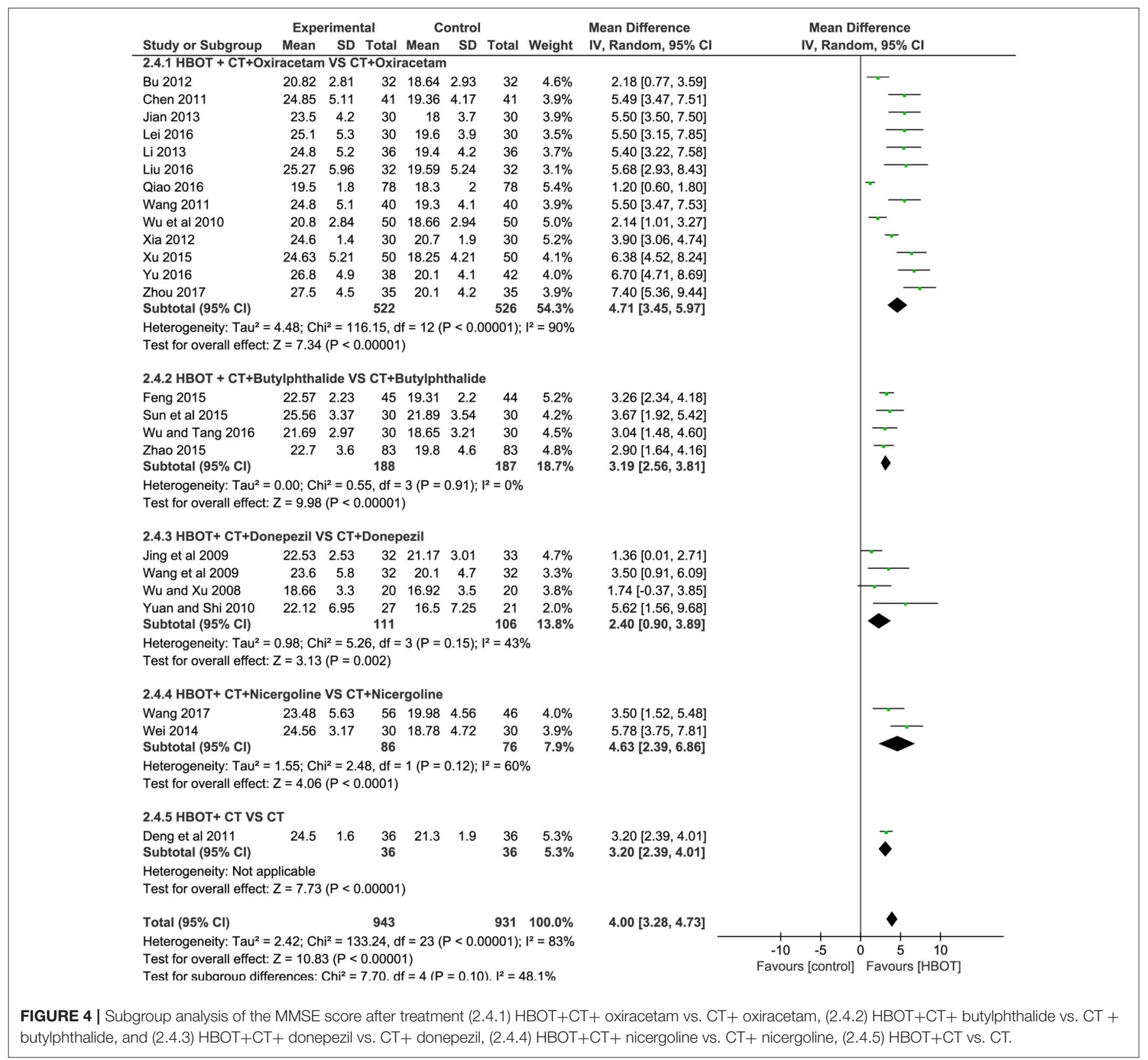

adverse reactions were mild, and no serious adverse reactions were reported. The result of meta-analysis indicated no statistic difference between the two groups, suggesting that the addition of HBOT did not increase adverse events. $(O R=0.85,95 \% C I=$ $0.26-2.78, P=0.79$ ), as shown in Figure 8.

\section{Sensitivity Analysis}

Stata 15.1 was employed for sensitivity analysis of the main outcomes, including TEF, MMSE score, ADL score, and BADL score. The results showed that removing any one study of each outcome had no significant effect on the overall results, indicating that the results of this meta-analysis were reliable, as shown in Figure 9.

\section{Publication Bias}

We used Stata15.1 software to detect the possible publication bias of primary outcomes, and trim and filling method was conducted to cope with striking publication bias if $P<0.05$. The result of Egger's test suggested that there was no publication bias in terms of TEF $(P>|t|=0.452,95 \% C I=-7.34$ to 3.59), ADL score $(P>|t|=0.869,95 \% C I=-1.62$ to 1.89$)$, BADL score $(P>|t|=0.571,95 \% C I=-9.92$ to 15.59$)$ and Adverse events $(P>|t|=0.056,95 \% C I=-0.16$ to 8.36$)$. For the MMSE score, significant publication bias was observed $(P>|t|=0.0001,95 \% C I=1.85-5.32)$ (Figure 10), and trim and filling method were used to evaluate the reliability of results affected by significant publication bias. After running the 


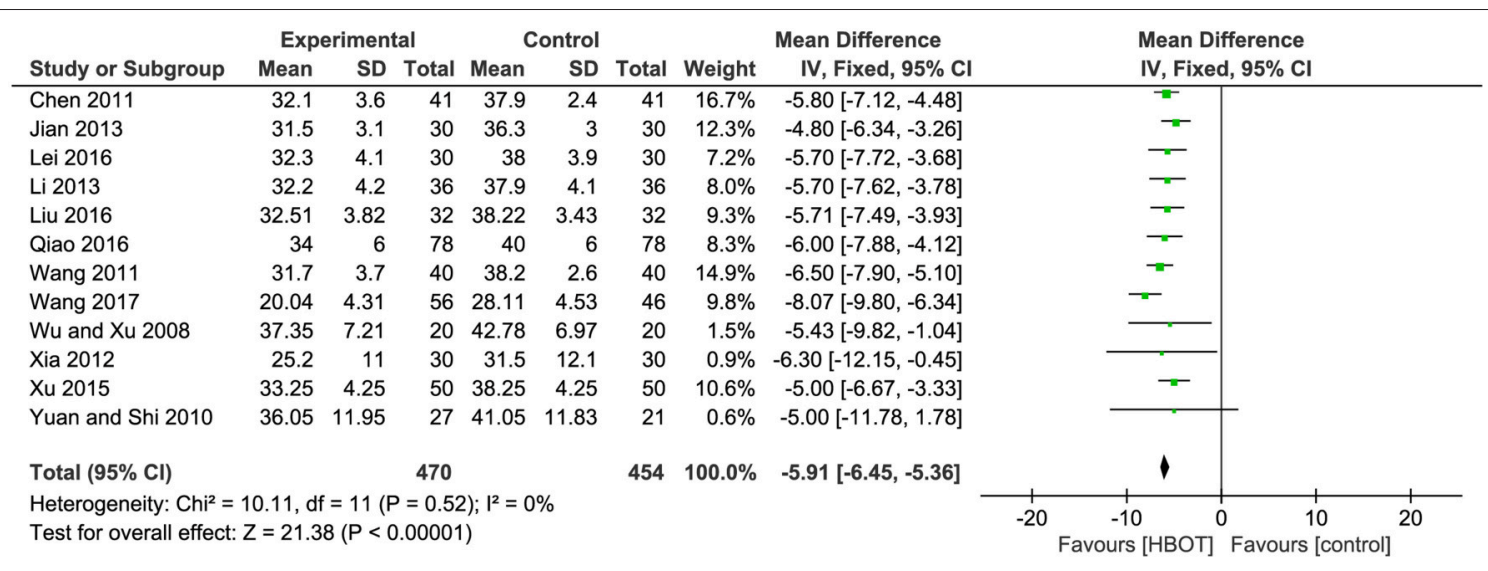

FIGURE 5 | Comparison of the ADL score after treatment between the HBOT group and the control group.

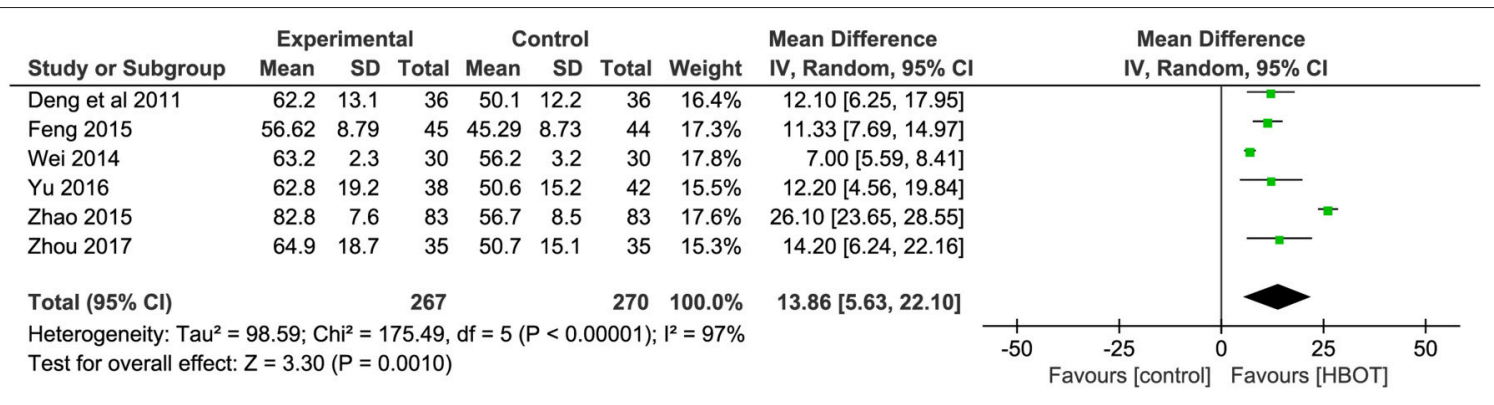

FIGURE 6 | Comparison of the BADL score after treatment between the HBOT group and control groups.

iterations, seven studies marked with squares in Figure 11 were filled. However, the $M D$ and $95 \% C I$ after trim and filling method $(M D=3.27 ; 95 \% C I=2.58-3.96 ; P<0.00001)$ was consistent with the previous result $(M D=4.00 ; 95 \% C I=3.28-4.73$; $P<0.00001)$, indicating that the result was stable without flip.

\section{DISCUSSION}

VD is a complex syndrome with varied pathogenesis, including infarct dementia, microvascular ischemia disease, poor perfusion and hemorrhage, mixed dementia and cerebral autosomal dominance, with subcortical infarcts and leukoencephalopathy (CADASIL) (O’Brien, 2006). Commonly used diagnostic criteria include NINDS-AIREN, ICD10, DSM-IV (Chen and Zhang, 2016). Computed tomography and magnetic resonance imaging can, to some extent, indicate the progression of strategic infarct dementia, lacunar infarction and periventricular damage of white matter. Due to a lack of ideal biochemical indexes in the clinical diagnosis of VD, there were no unified clinical diagnostic criteria worldwide, and pathological examination was the only gold standard. Generally, demographic factors, genetic factors, general vascular risk factors and stroke-related factors play an important role leading to VD. A systematic review found that the first stroke of patients caused 10\% VD, and at least $33.3 \%$ of $\mathrm{VD}$ resulted from palindromic stroke. However, current treatments focusing on reducing modifiable risk factors and neuroprotection are unsatisfactory. Therefore, finding clinically effective complementary therapies with lower adverse events can enhance the therapeutic efficacy of conventional therapies in treating VD.

HBOT, as an adjuvant treatment, has been widely used in cerebral injury and has shown great effects on reducing the disability rate and improving the cure rate. Because Hypoxia is one of the major pathological factors that leads to neuronal cell injury, HBOT achieves physiologic effects by increasing the oxygen level, raising oxygen tension, decreasing intracranial pressure and relieving brain edema. At the cellular level, animal studies suggested that HBOT decreased COX-2 mRNA and protein levels, and inhibited COX-2 overexpression in rats with cerebral ischemia (Yin et al., 2002). Additionally, the neuroprotection of HBOT is also associated with antioxidant effects and the reduction of apoptosis related to reducing the caspase-3 expression and activity (Yin et al., 2003; Calvert et al., 2004). A 2012 Cochrane system review was undertaken to address the efficacy and safety of HBOT for VD and concluded that the addition of HBOT for VD appeared to be more effective than the controls. However, the evidence obtained by them was probably unpersuasive because only one trial with 64 patients was analyzed in their review. Another systematic review (Chen and Zhang, 2016) conducted in China in 2016, demonstrated that applying HBOT as an adjunctive therapy strikingly improved the MMSE, ADL and HDS scores in VD patients. Despite the 
A

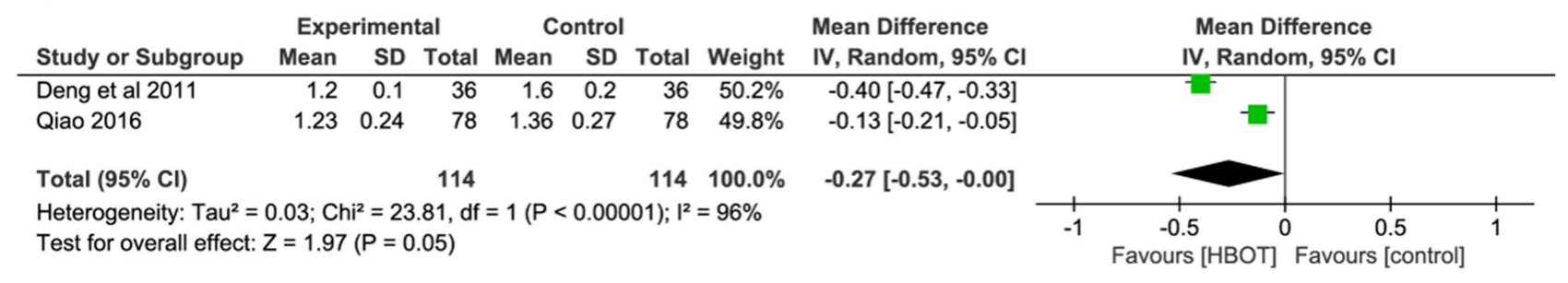

B

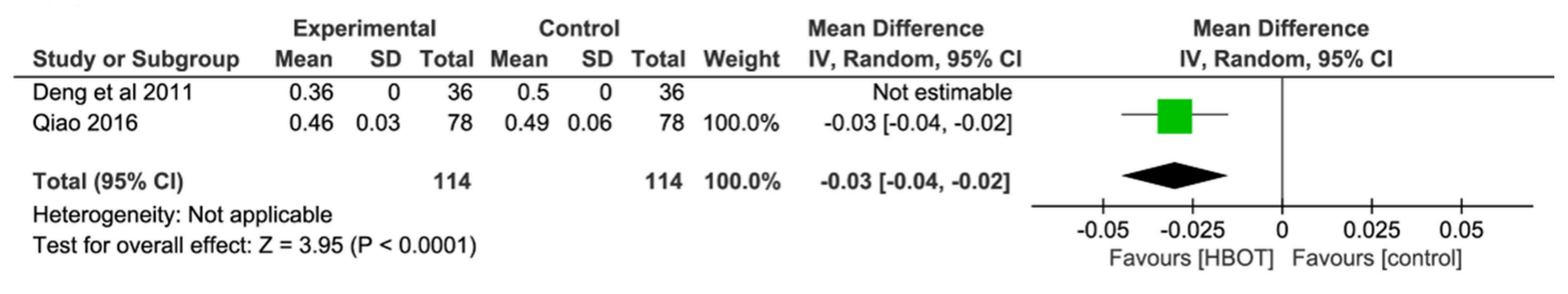

C

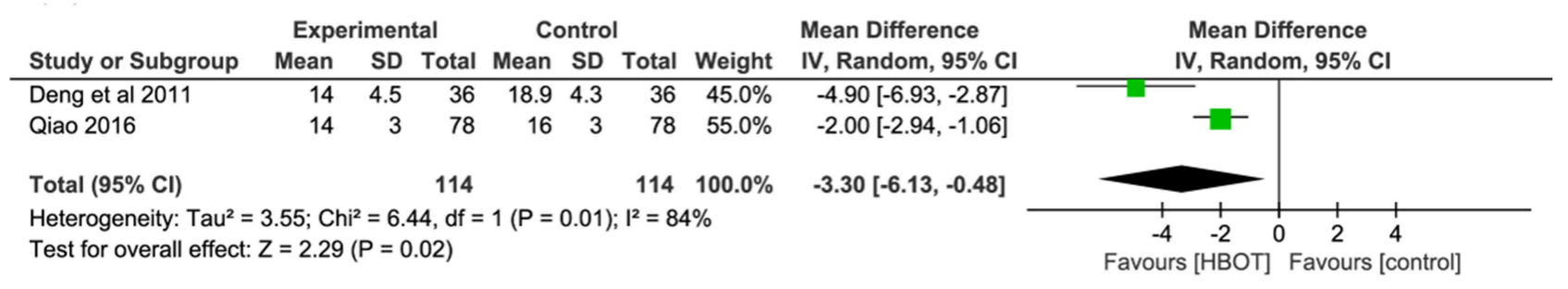

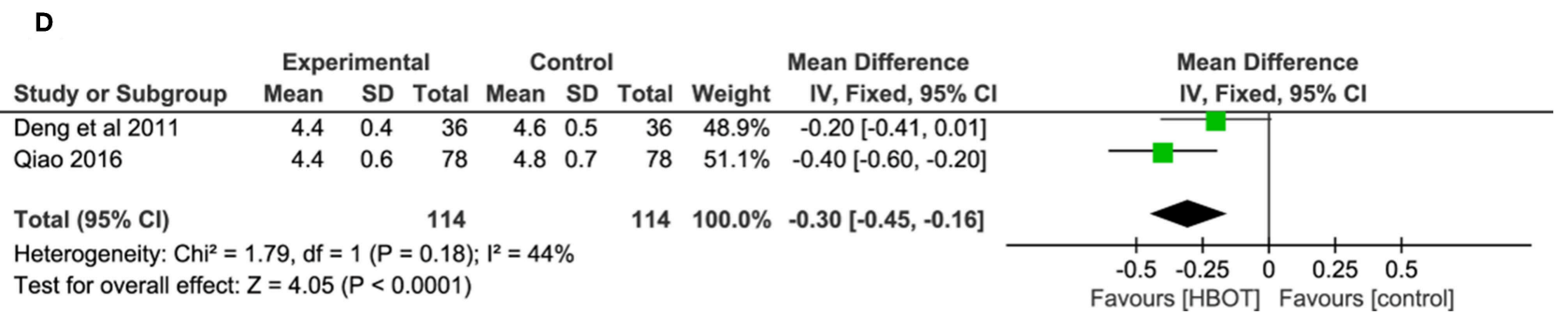

FIGURE 7 | Comparison of the Hemorheology after treatment between the HBOT group and control groups. (A) Plasma viscosity, (B) hematocrit value, (C) erythrocyte sedimentation rate (ESR), and (D) fibrinogen.

positive findings, their conclusion was not dependable because some studies included in their review were seriously flawed. For instance, there was a significant difference in the ADL score before intervention between the HBOT and CT groups in one of the included studies (Song, 2012), and the data from another study included in their review were obviously inconsistent (Bao and Zhong, 2007). Moreover, because only two of the 16 studies reported the adverse events, the conclusion regarding safety assessment of HBOT for VD was unconvincing. Therefore, we rigorously performed an updated systematic review to assess the effectiveness and safety of HBOT.

This updated meta-analysis assessed the evidence from 25 RCTs with a total of $1,954 \mathrm{VD}$ patients randomized to receive additional HBOT or CT between 2008 and 2017. The main results included the following: (1) MMSE score were strikingly high in the HBOT group when compared with the CT group, (2) ADL score significantly favored the HBOT group compared with the CT group, (3) for the BADL, patients in the HBOT group benefited more than those in the CT group, (4) compared with patients treated with $\mathrm{CT}$, the addition of $\mathrm{HBOT}$ resulted in a striking improvement in the TEF, (5) the HBOT group also showed additional benefits for improving hemorheology (plasma viscosity, hematocrit value, erythrocyte sedimentation rate and fibrinogen), and (6) the results across various subgroups were highly in agreement, and the benefits of HBOT were significant. No statistically significant difference was found between the HBOT and CT groups regarding the adverse events rate. 


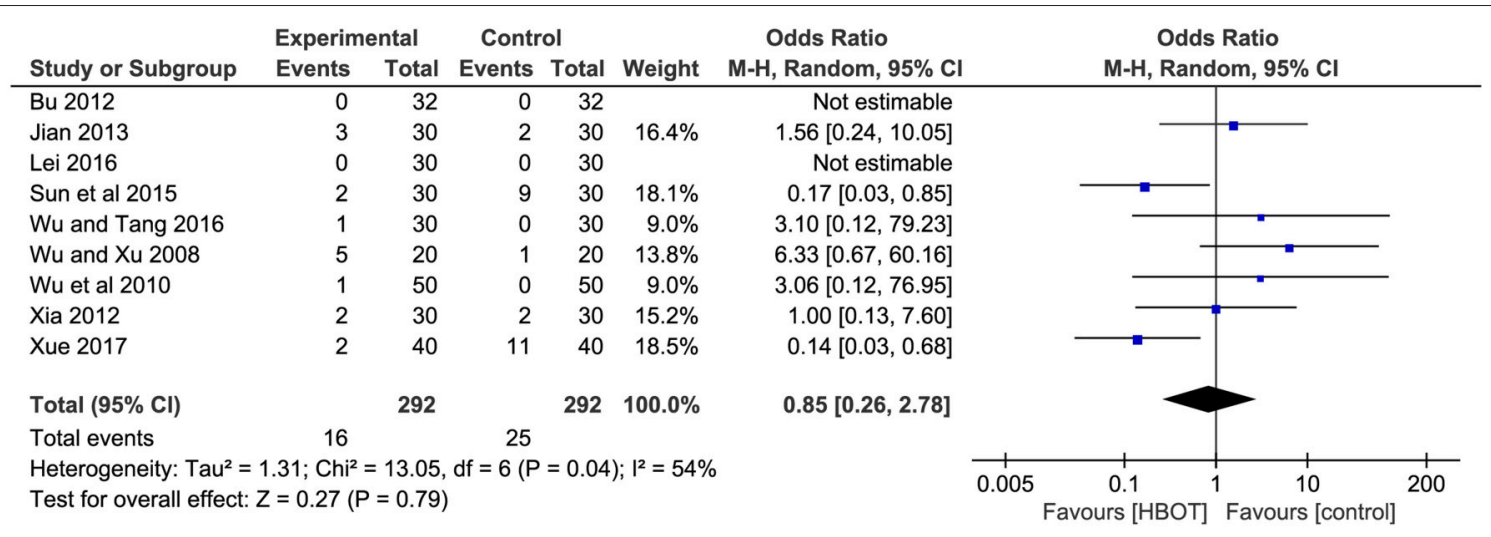

FIGURE 8 | Comparison of the adverse events after treatment between the HBOT group and control groups.

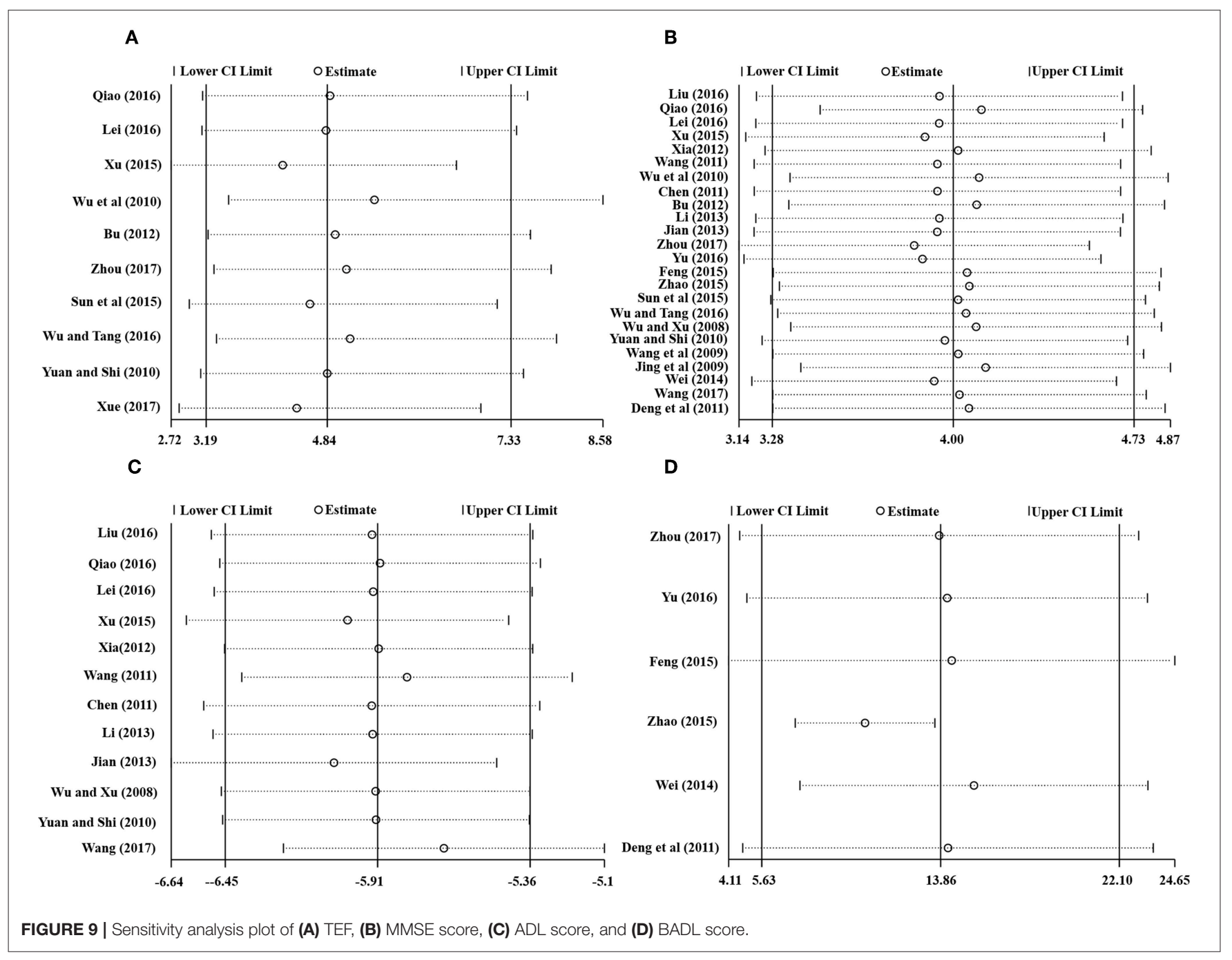

\section{Subgroup Analysis}

To determine the best treatment strategy of HBOT, subgroup analysis of main outcomes was introduced according to the daily oxygen intake and treatment duration. The results showed a higher effect size value and narrower confidence interval of the "7-8 weeks" group than that of the " $3-4$ weeks" and 

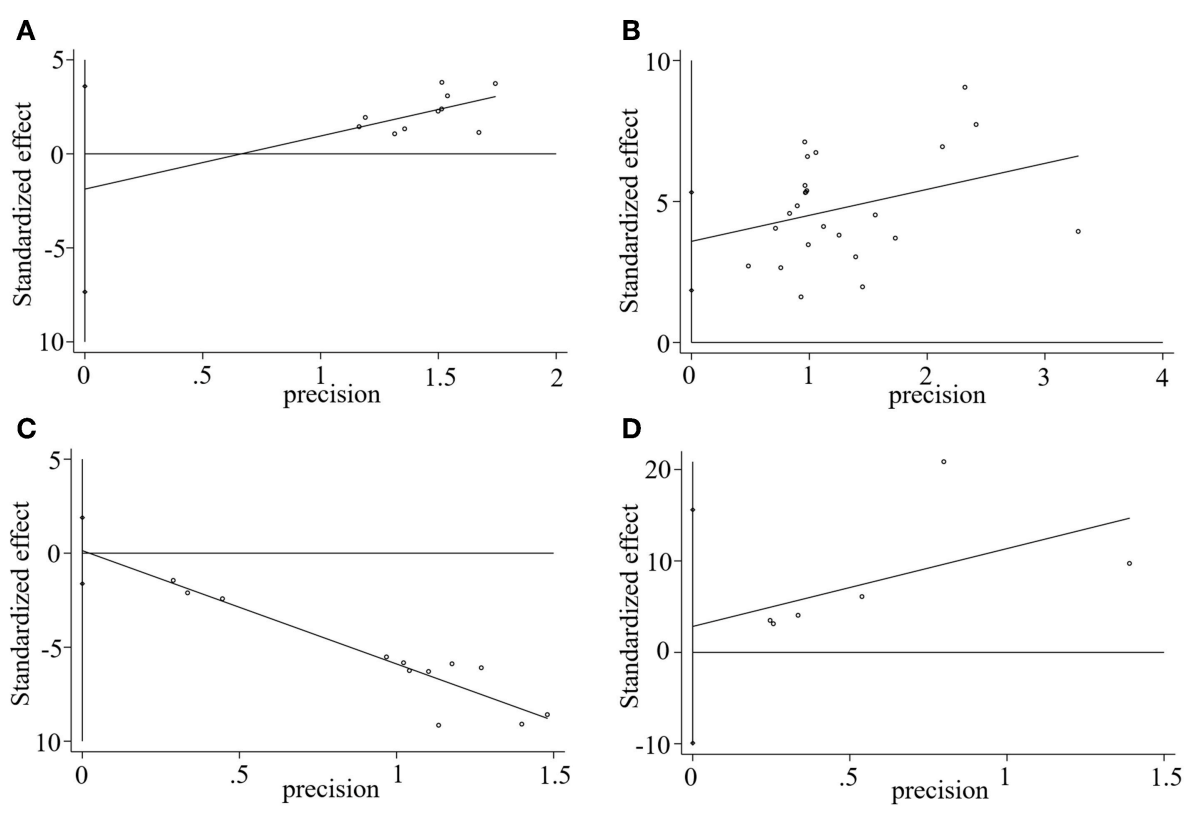

FIGURE 10 | Egger's publication bias plot of (A) TEF, (B) MMSE score, (C) ADL score, and (D) BADL score.

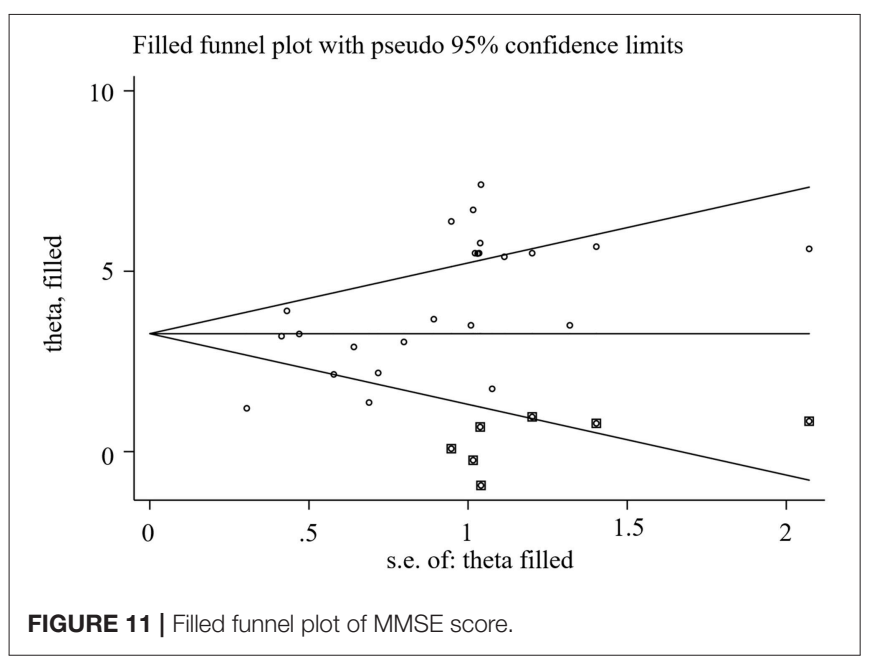

"12-16 weeks" group, which indicated that "7-8 weeks" of treatment duration bring the maximum therapeutic effect to the VD patients, and the results are more precise and reliable, especially subgroup analysis of ADL. Subgroup analysis of daily oxygen intake suggested that there was a significant difference between the 60 and $120 \mathrm{~min}$ groups. Despite the significant difference favoring the $60 \mathrm{~min}$ group, it is unreasonable to conclude that $60 \mathrm{~min}$ of HBOT was more effective than $120 \mathrm{~min}$ because the number of studies between subgroups varied widely. However, considering the compliance and medical burden of patients and the fact that 60 min of oxygen inhalation was sufficiently effective, 60 min of daily HBOT with 7-8 weeks of treatment duration was recommended for the treatment of VD, as shown in Table 3.

\section{Implications for Practice}

Before we conducted this review, several studies had demonstrated the therapeutic effect of HBOT for treating brainrelated diseases. An earlier animal experiment suggested that HBOT significantly improved learning, memory and recovery of blood perfusion in rats with VD by raising neurogenesis and cerebral blood flow in piriform cortex (Zhang et al., 2010). Then, a meta-analysis (Cui et al., 2017) relating to an intracerebral hemorrhage (ICH) animal model indicated that the HBOT group significantly reduced the brain water content (BWC) and improved the neuro-behavioral outcome (NO). In addition, another review including 51 trials for the animal experiment also showed that HBOT significantly improved neurological function and reduced the infarct size by $32 \%$ when compared with the CT group, and the mortality was $8.3 \%$ lower than that of the CT group (Xu et al., 2016). Almost at the same time, (Wang et al., 2016) reported that HBOT significantly improved the Glasgow coma scale $(M D=3.13,95 \%$ CI 2.34-3.92, $P<0.001)$, Glasgow outcome score of patients with traumatic brain injury $(O R=3.78,95 \% C I 1.23-11.63, P=0.020)$, and the overall mortality of HBOT group was strikingly lower than the CT group $(O R=0.32,95 \% C I 0.18-0.57, P<0.001)$. Compared with the previous two reviews, this meta-analysis first reported the effects of HBOT on hemorheology, which further explained the neuroprotective mechanism of HBOT. However, only two studies reported the hemorheology data, and most studies lacked data on degenerative cellular and molecular parameters such as apoptosis, cell death, inflammation and amyloid accumulation. Therefore, the mechanism of HBOT warrants further investigation. On the whole, the available evidence obtained from our review showed that application of HBOT as adjuvant therapy has additional benefits on VD patients and is 
generally safe. Furthermore, our findings are consistent with previous reports, which provides important clinical evidence for clinicians.

\section{Limitations}

Several limitations should be highlighted in our meta-analysis. First, we only searched the main English and Chinese databases. Therefore, some studies meeting our inclusion criteria published in other languages or databases may have been excluded. All included trials declared randomization, but only three studies described a specific randomization method. Blinded assessments were not detailed in all included documents, which may have exerted a potential impact on the objectivity of VD outcomes. Second, the inclusion criteria of these studies had small sample sizes with low-quality designs, which may give rise to overvaluing the benefit of HBOT. Additionally, there may be a certain degree of selective reporting bias because most studies had not been officially registered. Third, only two eligible studies reported the hemorheology. Fourth, 13 papers did not mention any information on adverse reactions. Thus, the safety assessment of additional HBOT in treating VD was unsatisfactory. Fifth, although the simple mental state examination scale (MMSE), first introduced by Folstein in 1975, has been widely used as a screening tool for dementia and mental disorders in hospitalized patients, the evaluation of therapeutic effect using MMSE was restricted because cross-cultural translation of MMSE is not reliable, and the translation is likely to be confusing due to language, script skills, culture and ethical norms, especially for patients with $<5$ years of education. However, although the deficiency listed above may undermine the quality of

\section{REFERENCES}

Bao, Z. Y., and Zhong, X. B. (2007). Clinical observation on the effect of hyperbaric oxygen on the treatment of vascular dementia. J. Gannan Med. Univ. 27, 532-533. doi: 10.3969/j.issn.1001-5779.2007.04.018

Barker, R., Ashby, E. L., Wellington, D., Barrow, V. M., Palmer, J. C., Kehoe, P. G., et al. (2014). Pathophysiology of white matter perfusion in Alzheimer's disease and vascular dementia. Brain 137, 1524-1532. doi: 10.1093/brain/awu040

$\mathrm{Bu}, \mathrm{G}$. W. (2012). Clinical observation of hyperbaric oxygen combined with oralacetam in the treatment of vascular dementia in 96 cases. J. Psychol. 80. doi: 10.3969/j.issn1007-8231.2012.10.082

Calvert, J. W., Zhou, C., Nanda, A., and Zhang, J. H. (2004). Effect of hyperbaric oxygen on apoptosis in neonatal hypoxia-ischemia rat model. J. Appl. Physiol. 96, 2072-2080. doi: 10.1152/japplphysiol.00630.2003

Chen, L. P., Wang, F. W., Zuo, F., Jia, J. J., and Jiao, W. G. (2011). Clinical research on comprehensive treatment of senile vascular dementia. J. Tradit. Chin. Med. 31, 178-181. doi: 10.1016/S0254-6272(11)60036-8

Chen, S. D. (2011). Clinical observation of hyperbaric oxygen combined with oralacetam in the treatment of vascular dementia in 41 cases. J. Med. Theor. Prac. 24, 1425-1426. doi: 10.3969/j.issn.1001-7585.2011.12.028

Chen, Y. Y., and Zhang, Y. D. (2016). Meta-analysis of randomized controlled trials of hyperbaric oxygen in treating vascular dementia. Chin. Modern Med. 23, 16-19. Available online at: http://www.wanfangdata.com.cn/details/detail. do?_type=perio\&id=jkr201629005

Christopher, L. (1994). Diagnostic and Statistical Manual of Mental Disorders (DSM-IV) $[M]$. 4th Edn. Washington, DC: America Phychiatric Association, 213-219.

Cui, H. J., He, H. Y., Yang, A. L., Zhou, H. J., Tang, T., and Luo, J. K. (2017). Hyperbaric oxygen for experimental intracerebral haemorrhage: Systematic review and stratified meta-analysis. Brain Injury 31, 456-465. doi: 10.1080/02699052.2017.1279752 evidence, the included trials are highly comparable, and the documents were selected in relatively strict inclusion criteria. Since the patients of selected studies were mainly from China, the conclusion of this meta-analysis is not applicable to other ethnic groups. Therefore, large sample trials with high-quality and welldesigned ethnic groups should be conducted in the future to provide more reliable evidence regarding the efficacy and safety of HBOT for VD.

\section{CONCLUSION}

The present evidence from this meta-analysis suggested that the addition of HBOT to standard conventional therapies for VD significantly improved the MMSE, ADL, BADL, hemorheology, and clinical efficacy. In view of the effectiveness and safety of HBOT, it is reasonable to recommend HBOT as a complementary therapy for the treatment of VD.

\section{AUTHOR CONTRIBUTIONS}

QY conceived this review and completed the manuscript. LL performed the literature searches electronically and manually. NY and DL performed study selection and data extraction. YY and SX assessed the risk of bias. YL and HC critically revised the paper.

\section{FUNDING}

This study was supported by Chinese Natural Sciences Foundation (No: 8140140928).

Deng, X. L., Wang, J., and Zhao, B. (2011). Effect of hyperbaric oxygen on cognition and activity of daily life and hemorheology of patients with vascular dementia. Chin. J. Rehabil. 26, 96-97. doi: 10.3870/zgkf.2011.02.007

Etherton-Beer, C. D. (2014). Vascular cognitive impairment in dementia. Maturitas 79, 220-226. doi: 10.1016/j.maturitas.2014.06.004

Feldmeier, J. J., and Undersea and Hyperbaric Medical Society (2003). Hyperbaric Oxygen (2003): Indications and Results: The Hyperbaric Oxygen Therapy Committee Report. Kensington, CA: Undersea and Hyperbaric Medical Society.

Feng, Y. H. (2015). Effects of butylphthalide combined with hyperbaric oxygen on cognitive ability in patients with post-stroke dementia. Chin. J. Pract. Neurol. Dis. 18, 121-122. doi: 10.3969/j.issn.1673-5110.2015. 24.087

Ferri, C. P., Prince, M., Brayne, C., Brodaty, H., Fratiglioni, L., Ganguli, M., et al. (2005). Global prevalence of dementia: a Delphi consensus study. Lancet 366, 2112-2117. doi: 10.1016/S0140-6736(05)67889-0

García-Covarrubias, L., and Cuauhtémoc Sánchez-Rodríguez, E. (2000). Hyperbaric oxygenation therapy, basic concepts. Gac. Med. Mex. 136, 45-56.

Gorelick, P. B., Scuteri, A., Black, S. E., Decarli, C., Greenberg, S. M., Iadecola, C., et al. (2011). Vascular contributions to cognitive impairment and dementia: A statement for healthcare professionals from the American Heart Association/American Stroke Association. Stroke 42, 2672-2713. doi: 10.1161/STR.0b013e3182299496

Hasnain, M., and Vieweg, W. V. (2014). Possible role of vascular risk factors in Alzheimer's disease and vascular dementia. Curr. Pharm. Des. 20, 6007-6013. doi: 10.2174/1381612820666140314153440

Jellinger, K. A. (2014). Pathogenesis and treatment of vascular rcognitive impairment. Neurodegener. Dis. Manag. 4, 471-490. doi: 10.2217/nmt.14.37

Jian, H. B. (2013). Clinical observation of hyperbaric oxygen combined with oralacetam in the treatment of vascular dementia. China Rural Health. 4, 6-7. Available online at: http://www.cqvip.com/QK/89405X/201303Z/47081513. html 
Jing, H. J., and Luo, S. P. (2009). Observation of hyperbaric oxygen combined with donepezil in the treatment of vascular dementia with aphasia. Pract. Gerontol. 23, 475-476. doi: 10.3969/j.issn.1003-9198.2009.06.026

Kalaria, R. N., Akinyemi, R., and Ihara, M. (2016). Stroke injury, cognitive impairment and vascular dementia. Biochim. Biophys. Acta. 1862, 915-925. doi: 10.1016/j.bbadis.2016.01.015

Kalaria, R. N., Maestre, G. E., Arizaga, R., Friedland, R. P., Galasko, D., Hall, K., et al. (2008). Alzheimer's disease and vascular dementia in developing countries: prevalence, management, and risk factors. Lancet Neurol. 7, 812-816. doi: $10.1016 / S 1474-4422(08) 70169-8$

Lei, X. D. (2016). Clinical observation on the curative effect of oralacetam combined with hyperbaric oxygen in the treatment of vascular dementia. Heilongjiang Med. Pharm. 39, 147-149. doi: 10.3969/j.issn.1008-0104.2016.01.068

Levine, D. A., and Langa, K. M. (2011). Vascular cognitive impairment: disease mechanisms and therapeutic implications. Neurotherapeutics 8, 361-373. doi: $10.1007 / \mathrm{s} 13311-011-0047-\mathrm{z}$

Li, W. (2013). Curative effect analysis of hyperbaric oxygen associated with oxiracetam on 36 eases of Vaseular dementia. China Med. Pharm. 4, 63-64. Available online at: http://www.cqvip.com/QK/97963A/201304/45198696.html

Liu, Z. Y. (2016). Clinical effect analysis of oralacetam combined with hyperbaric oxygen in the treatment of vascular dementia. China Med. Device Inform. 22, 87-88. doi: 10.15971/j.cnki.cmdi.2016.18.043

O’Brien, J. T. (2006). Vascular cognitive impairment. A. J. Geriatr. Psychiatry 14, 724-733. doi: 10.1097/01.JGP.0000231780.44684.7e

O'Brien, J. T., and Markus, H. S. (2014). Vascular risk factors and Alzheimer's disease. BMC Med. 12:218. doi: 10.1186/s12916-014-0218-y

O'Brien, J. T., and Thomas, A. (2015). Vascular dementia. Lancet 386, 1698-1706. doi: 10.1016/S0140-6736(15)00463-8

Qian, C. Y., lv, C. Z., and Wang, X. D. (2002). Draft diagnostic criteria for vascular dementia. Chin. J. Neurol. 35, 87-93. doi: 10.3760/j.issn:1006-7876.2002.04.036

Qiao, Y. (2016). Study on the clinical effect of oralacetam combined with hyperbaric oxygen in the treatment of vascular dementia. China J. Pharm. Econ. 11, 46-48. doi: 10.12010/j.issn.1673-5846.2016.06.014

Robertson, P. W., and Hart, B. B. (1999). Assessment of tissue oxygenation. Respir Care Clin N Am. 5, 221-263.

Sahathevan, R., Brodtmann, A., and Donnan, G. A. (2012). Dementia, stroke, and vascular risk factors; a review. Int. J. Stroke 7, 61-73. doi: $10.1111 /$ j.1747-4949.2011.00731.x

Sanchez, E. C. (2013). Mechanisms of action of hyperbaric oxygenation in stroke: a review. Crit. Care Nurs. Q. 36, 290-298. doi: 10.1097/CNQ.0b013e318294e9e3

Song, Y. H. (2012). Study on the clinical effect of oralacetam combined with hyperbaric oxygen in the treatment of vascular dementia in 63 cases. Healthy People. 6, 5-7. Available online at: http://www.wanfangdata.com.cn/details/ detail.do?_type=perio\&id=QK201201786706

Sorrentino, G., Migliaccio, R., and Bonavita, V. (2008). Treatment of vascular dementia: the route of prevention. Eur. Neurol. 60, 217-223. doi: $10.1159 / 000151696$

Sun, W., Yang, J. B., Xu, J. F., Zhang, Q., Zou, Q., Meng, X. L., et al. (2015). Clinical effect of Butylphthalide soft capsules combined with hyperbaric oxygenation on senile vascular dementia. J. Pract. Cardiovasc. Cerebrovasc. Dis. 23, 62-64. doi: 10.3969/j.issn.1008-5971.2015.09.019

Wang, F., Wang, Y., Sun, T., and Yu, H. L. (2016). Hyperbaric oxygen therapy for the treatment of traumatic brain injury: a meta-analysis. Neurol. Sci. 37, 693-701. doi: 10.1007/s10072-015-2460-2

Wang, Q. P. (2017). Effect of nicergoline combined with hyperbaric oxygen therapy on cognitive function in patients with vascular dementia. China Health Stand. Manag. 8, 63-64. doi: 10.3969/j.issn.1674-9316.2017.15.036

Wang, S. L., and Zhai, L. H. (2011). Clinical observation of hyperbaric oxygen combined with oralacetam in the treatment of vascular dementia in 40 cases. Guide China Med. 9, 312-313. doi: 10.3969/j.issn.1671-8194.2011.32.243

Wang, S. P., Tao, Z., Ding, S. J., Cheng, J. C., Yu, B. S., and Wang, Y. Q. (2009). Hyperbaric oxygen combined with donepezidine in the treatment of vascular dementia. Chin. J. Phys. Med. Rehabil. 31, 478-480. doi: 10.3760/cma.j.issn.0254-1424.2009.07.016

Wei, N. L. (2014). Study on the effects of nicergoline combined with hyperbaric oxygen on cognitive function in patients with vascular dementia. Chongqing Med. 43, 4529-4531. doi: 10.3969/j.issn.1671-8348.2014.33.040
Wu, H. L., and Tang, Y. Y. (2016). Therapeutic effect of butylphthalide combined with hyperbaric oxygen in the treatment of vascular dementia. Modern Med. Health. 32, 3696-3697. doi: 10.3969/j.issn.1009-5519.2016.23.046

$\mathrm{Wu}, \mathrm{J}$. Y., and $\mathrm{Xu}$, P. (2008). Effects of donepezil hydrochloride plus hyperbaric oxygen on cognitive function and activites of daily life ability in patients with mild and moderate vascular dementia. Chin. J. Geriatr. Care 6, 35-37. doi: 10.3969/j.issn.1672-4860-B.2008.05.015

$\mathrm{Wu}$, Y., Liang, Y., and Huang, D. D. (2010). Clinical observation on the curative effect of oralacetam combined with hyperbaric oxygen in the treatment of vascular dementia. Chin. J. Pract. Neurol. Dis. 13, 13-14. doi: 10.3969/j.issn.1673-5110.2010.07.006

Xia, X. Y. (2012). Observation on the curative effect of hyperbaric oxygen on vascular dementia. Asia Pacific Tradit. Med. 8, 84-85. doi: 10.3969/j.issn.1673-2197.2012.01.041

Xiao, Y. S., Wang, J., Jiang, S., and Luo, H. Y. (2012). Hyperbaric oxygen therapy for vascular dementia Review. Cochrane Database Syst. Rev. 11:CD009425. doi: 10.1002/14651858.CD009425.pub2

$\mathrm{Xu}, \mathrm{M}$. (2015). Analyze the application of oralacetam and hyperbaric oxygen in the treatment of vascular dementia. China Contin. Med. Educ. 7, 190-191. doi: 10.3969/j.issn.1674-9308.2015.30.141

Xu, Y., Ji, R., Wei, R., Yin, B., He, F., and Luo, B. (2016). The efficacy of hyperbaric oxygen therapy on middle verebral artery occlusion in animal studies: a metaanalysis. PLoS ONE. 11:e0148324. doi: 10.1371/journal.pone.0148324

Xue, B. Q. (2017). Clinical effect of butylphthalide combined with hyperbaric oxygen in the treatment of senile vascular dementia. China Prescript. Drug 15, 66-67. Available online at: http://www.wanfangdata.com.cn/details/detail. do?_type $=$ perio\&id $=$ zgcfy 201710047

Yates, K. F., Sweat, V., Yau, P. L., Turchiano, M. M., and Convit, A. (2012). Impact of metabolic syndrome on cognition and brain: a selected review of the literature. Arterioscler. Thromb. Vasc. Biol. 32, 2060-2067. doi: 10.1161/ATVBAHA.112.252759

Yin, D., Zhou, C., Kusaka, I., Calvert, J. W., Parent, A. D., Nanda, A., et al. (2003). Inhibition of apoptosis by hyperbaric oxygen in a rat focal cerebral ischemic model. J. Cereb. Blood Flow Metab. 23, 855-864. doi: 10.1097/01.WCB.0000073946.29308.55

Yin, W., Badr, A. E., Mychaskiw, G., and Zhang, J. H. (2002). Down regulation of COX-2 is involved in hyperbaric oxygen treatment in a rat transient focal cerebral ischemia model. Brain Res. 926, 165-171. doi: 10.1016/S0006-8993(01)03304-2

Yu, Y. (2016). Analysis of the clinical effect of oralacetam combined with hyperbaric oxygen in the treatment of vascular dementia. China. Prac. Med. 11, 168-169. doi: 10.14163/j.cnki.11-5547/r.2016.19.121

Yuan, L. J., and Shi, X. Y. (2010). Clinical study on the treatment of senile dementia by hyperbaric oxygen combined with donepezil hydrochloride. Anhui Med. J. 31, 889-892. doi: 10.3969/j.issn.1000-0399.2010.08.09

Zhang, T., Yang, Q. W., Wang, S. N., Wang, J. Z., Wang, Q., Wang, Y., et al. (2010). Hyperbaric oxygen therapy improves neurogenesis and brain blood supply in piriform cortex in rats with vascular dementia. Brain Injury 24, 1350-1357. doi: 10.3109/02699052.2010.504525

Zhao, P. X. (2015). Therapeutic effect of butylphthalide soft capsule combined with hyperbaric oxygen in the treatment of vascular dementia. Public Med. Forum Mag. 19, 610-611.

Zhou, A. H. (2017). Clinical study on the effect of oralacetam combined with hyperbaric oxygen in the treatment of vascular dementia. World Clin. Med. 11, 53-55. Available online at: http://www.wanfangdata.com.cn/details/detail. do?_type=perio\&id=zgcfy 201710047

Conflict of Interest Statement: The authors declare that the research was conducted in the absence of any commercial or financial relationships that could be construed as a potential conflict of interest.

Copyright (0) 2019 You, Li, Xiong, Yan, Li, Yan, Chen and Liu. This is an open-access article distributed under the terms of the Creative Commons Attribution License (CC $B Y)$. The use, distribution or reproduction in other forums is permitted, provided the original author(s) and the copyright owner(s) are credited and that the original publication in this journal is cited, in accordance with accepted academic practice. No use, distribution or reproduction is permitted which does not comply with these terms. 